\title{
Model Establishment and Loss Analysis of Transmission Line
}

\author{
Liu Hongzheng ${ }^{1, a}$, Yuan Haiyan,,a, Wu Linyann, ${ }^{2, b}$, Sui Shougang ${ }^{2, b}$, Wang \\ $\mathrm{TaO}^{2, \mathrm{~b}}$, Shi Zhen ${ }^{2, \mathrm{~b}}$ \\ 'State Grid Shandong Electric Power Research Institute, China
}

${ }^{2}$ Robotics and Intelligent Systems Research Institute of Shandong Jianzhu University, China a260347573@qq.com, bszxx307@126.com

\begin{abstract}
Keywords: Transmission line models; EMTP; Lightning protection performance.
Abstract. The paper discusses the influence of different transmission line models on lightning protection performance of transmission line under different circumstances by studying the mathematical model and calculation method of transmission lines. And the paper uses EMTP method to compare the actual calculation results and the actual results. The measurement errors introduced in different transmission lines model under different conditions are calculated to determine which model is more concise and effective within acceptable error range. And these results provide a strong theoretical basis for lightning protection performance analysis of transmission lines.
\end{abstract}

\section{Introduction}

As an important power element, transmission line plays an important role in many fields, such as power system calculation and so on. There are many mathematical models of transmission lines, from simple lumped parameter models to complicated distributed parameter models. Among them, the lumped parameter model includes R-L model, PI model and multilevel PI model, and the distributed parameter model includes lossless model, distortionless model and frequency dependent model. In the power system, the choice of different transmission line model directly affects the reliability and accuracy of the calculation results.

Inductance model of tower is the traditional tower equivalent inductance model our country adopts as the lightning protection method of the current regulations. It is a theoretical study of the single return transmission line, after the statistical summary of operation. The calculation results by using concentrated inductance to simulate the wave process on the tower are often too conservative, resulting in excessive investment in the line. Therefore, in the establishment of the tower equivalent model, this paper takes the multi wave impedance model as an example and analyzes the loss analysis method.

\section{Multi wave impedance model}

Although the single wave impedance model is better than the lumped inductance model, if the tower is regarded as such a simple structure, it is too simplified and can not be applied to the actual tower with complex structure and high height. So this paper proposes a new tower model - multi wave impedance model, this model not only takes into account the wave on the tower of the road, but also taking into account the structure of the tower itself, changes in different height to ground capacitance. So the result can be more in line with the results of wave process of high tower.

The foundation of the multi wave impedance model is based on the concept that the wave impedance at different heights of the vertical conductor is different, as shown in Figure 1. When the traveling wave arrives the radius $x_{1}=C t_{1}$ covered area, the cost time ${ }^{t=t_{1}}$. The wave cannot reach any other region, so the wave impedance $Z\left(x_{1}\right)$ of this vertical conductor from the starting point ${ }^{x_{1}}$, which can be obtained by geometric and physical parameters in the ${ }^{x_{1}}$ region. Thus it may be known the wave impedance of the vertical conductor is different from the starting point. We can divide these vertical conductor into sections, and each section can calculate a wave impedance, which is used as the 
theoretical basis to describe the multi wave impedance vertical conductor (lightning tower, etc.). In theory, the smaller the length of our segmentation, the more accurate the result is.

From the characteristics of the wave impedance, it is known that the wave impedance of the vertical cylinder depends only on the radius and height of the cylinder, so that the following empirical formula can be used to describe the wave impedance of the single vertical conductor. $r$ and $h$ are the radius and height perpendicular to the cylinder. In the formula, $\mathrm{C}$ means the wave velocity, $\mathrm{t}$ means the cost time, $\mathrm{x}$ means the distance to the origin, $\mathrm{r}$ means the travel time of the wave from tower top to column foot.

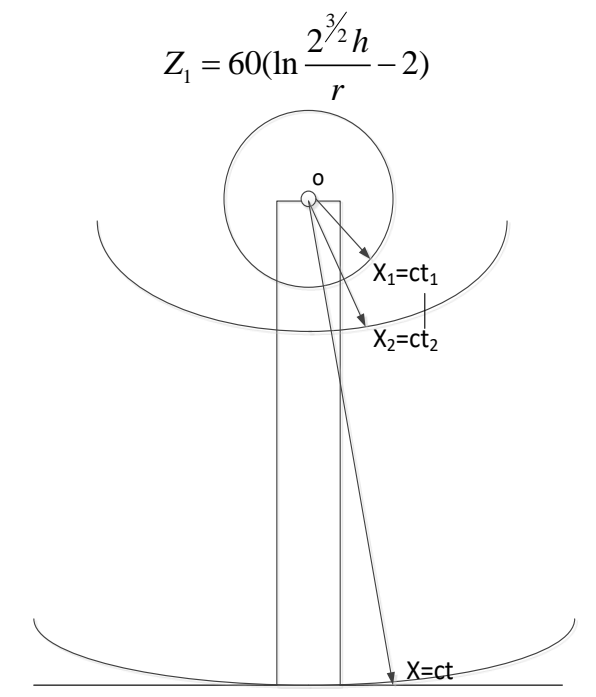

Figure 1. The motion of waves on a vertical conductor

That is, a multi conductor system contains several parallel cylinders, and the total wave impedance of the system consisting of $\mathrm{N}$ parallel cylinders is

$$
Z_{T, n}=\frac{1}{n}\left(Z_{T, 11}+Z_{T, 12}+\ldots .+Z_{T, 1 \mathrm{n}}\right)
$$

$\mathrm{n}$ is the number of cylinders, $Z_{T, 11}$ is the self impedance of the first cylinder; $Z_{T, 1 \mathrm{k}}(k=2, \cdots, n)$ is the mutual impedance between the first and the $\mathrm{K}$ root.

Assuming that equation (1) is valid for the system, then the mutual wave impedance $Z_{T, k k}$ and the mutual wave impedance $Z_{T, k l}$ between the root of the first and the $\mathrm{K}$ root can be expressed as

$$
\begin{aligned}
Z_{T, k k} & =60\left(\ln \frac{2^{3 / 2} h}{r}-2\right) \\
Z_{T, k l} & =60\left(\ln \frac{2^{3 / 2} h}{r}-2\right)
\end{aligned}
$$

In view of the fact that the tower is at most a 4 conductor system, the paper gives the formulae for calculating the wave impedance of the 2, 3 and more than 4 conductor systems

$$
Z_{T, k l}=60\left(\ln \frac{2^{3 / 2} h}{r_{e}}-2\right)
$$

In the formula, $r_{e}$ is the equivalent radius of a multi conductor system, which can be calculated by the following formula

$$
r_{e}=\left\{\begin{array}{c}
r^{1 / 2} R^{1 / 2}(n=2) \\
r^{1 / 3} R^{2 / 3}(n=3) \\
2^{1 / 8} r^{1 / 4} R^{3 / 4}(n=4)
\end{array}\right.
$$

In the formula, $\mathrm{R}$ is the distance between adjacent conductors of a multi conductor system consisting of parallel conductors. 

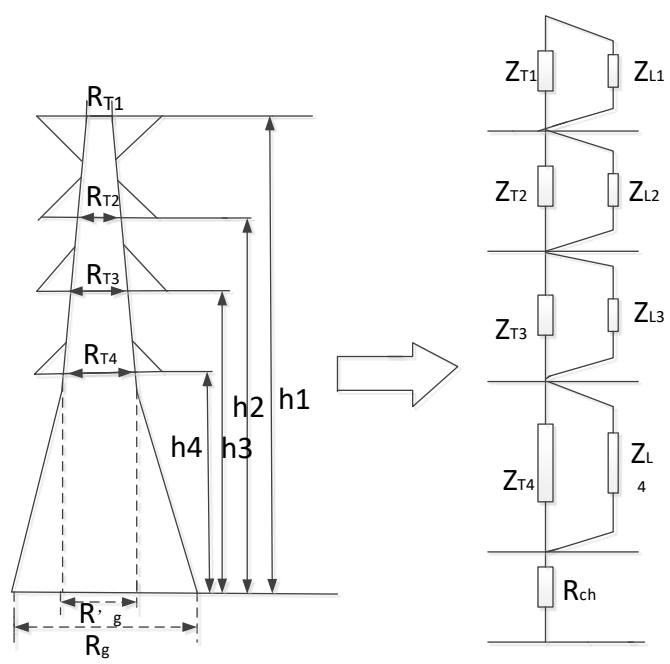

Figure 2. Multi wave impedance model

In a multi conductor system with a non parallel conductor, the equivalent radius formula is derived from the empirical formula of the measurement results:

$$
\begin{aligned}
& r=r_{T}^{1 / 3} r_{B}^{2 / 3} \\
& R=R_{T}^{1 / 3} R_{B}^{2 / 3}
\end{aligned}
$$

The towers can be divided into main supports and supports, each of which is assumed to be uniformly distributed, and their wave impedance is calculated by their own size and geometry(figure 2).

\section{Ground loss model}

When the overhead earth wire is in normal operation, it has electrostatic induction and electromagnetic coupling with the transmission wire, so that the overhead ground wire generates an induced voltage. The induced current and loss induced by electrostatic induction on overhead earth wire are very small and negligible. The earth circulation on the open ground line is mainly affected by the electromagnetic induction voltage. When the transmission line is in normal operation, the voltage and current on the line generate electromagnetic field around the conductor. Electromagnetic induction and electrostatic coupling are generated between the ground wire and the wire, it will induce electromagnetic induction electromotive force and electrostatic induction electromotive force along the ground line. If the ground wire is grounded by tower, a considerable induced circulation will occur in the loop of ground wire - ground wire and ground wire - ground, and a large amount of electric energy is wasted. Especially in unbalanced wire transposition, three-phase load asymmetry or line failure, the induced circulation is larger. At present, in China, the $220 \mathrm{kV}$ and above neutral point grounding system usually adopts two ground symmetrical erection methods, one uses common ground wire (GJ ground wire), and one uses OPGW. In the past, due to thelow voltage level of the transmission line, small transmission capacity and small load current, the total loss of ground induction circulating current was not large enough, so it received little attention. With the increase of power demand, the voltage level increases, the transmission capacity increases, though the proportion of ground induced circulating current loss is not large, the total amount is increasing, which results in the increase of operating cost. The electric energy loss of ground wire is closely related to the grounding mode of ground wire and the operation of the circuit.

\section{Ground loss calculation method}

During the operation of the transmission line, the conductor has a certain voltage. through a certain load current, a strong electromagnetic field is formed around it. The overhead ground wire is in the electromagnetic field, and the induction voltage is generated on it. Induction voltage and current can be divided into two parts: electromagnetic induction and static induction. Electrostatic induction is mainly due to the coupling capacitance between wires. At present, the overhead ground wire has at 
least one grounding point in the power system, and the electrostatic induction phenomenon can be ignored.

Magnetic coupling effects between electromagnetic circuits, Electromagnetic induction matrix is $[U]=[Z] \cdot[I]$. Applied to the same tower dual transmission line system, the relationship between the induced voltage and the current on the earth wire is shown in equation (8).

$$
\left[\begin{array}{c}
U_{1} \\
U_{2} \\
U_{A 1} \\
U_{B 1} \\
U_{C 1} \\
U_{A 2} \\
U_{B 2} \\
U_{C 2}
\end{array}\right]=\left[\begin{array}{cccccccc}
Z_{11} & Z_{12} & Z_{1, A 1} & Z_{1, B 1} & Z_{1, C 1} & Z_{1, A 2} & Z_{1, B 2} & Z_{1, C 2} \\
Z_{21} & Z_{22} & Z_{2, A 1} & Z_{2, B 1} & Z_{2, C 1} & Z_{2, A 2} & Z_{2, B 2} & Z_{2, C 2} \\
Z_{A 1,1} & Z_{A 1,2} & Z_{A 1, A 1} & Z_{A 1, B 1} & Z_{A 1, C 1} & Z_{A 1, A 2} & Z_{A 1, B 2} & Z_{A 1, C 2} \\
Z_{B 1,1} & Z_{B 1,2} & Z_{B 1, A 1} & Z_{B 1, B 1} & Z_{B 1, C 1} & Z_{B 1, A 2} & Z_{B 1, B 2} & Z_{B 1, C 2} \\
Z_{C 1,1} & Z_{C 1,2} & Z_{C 1, A 1} & Z_{C 1, B 1} & Z_{C 1, C 1} & Z_{C 1, A 2} & Z_{C 1, B 2} & Z_{C 1, C 2} \\
Z_{A 2,1} & Z_{A 2,2} & Z_{A 2, A 1} & Z_{A 2, B 1} & Z_{A 2, C 1} & Z_{A 2, A 2} & Z_{A 2, B 2} & Z_{A 2, C 2} \\
Z_{B 2,1} & Z_{B 2,2} & Z_{B 2, A 1} & Z_{B 2, B 1} & Z_{B 2, C 1} & Z_{B 2, A 2} & Z_{B 2, B 2} & Z_{B 2, C 2} \\
Z_{C 2,1} & Z_{C 2,2} & Z_{C 2, A 1} & Z_{C 2, B 1} & Z_{C 2, C 1} & Z_{C 2, A 2} & Z_{C 2, B 2} & Z_{C 2, C 2}
\end{array}\right]\left[\begin{array}{c}
I_{1} \\
I_{2} \\
I_{A 1} \\
I_{B 1} \\
I_{C 1} \\
I_{A 2} \\
I_{B 2} \\
I_{C 2}
\end{array}\right]
$$

In equation (8), $[\mathrm{U}]$ is the earth wire voltage drop matrix, and $[\mathrm{Z}]$ is the earth wire full impedance matrix, the diagonal element $Z_{i i}$ represents the self impedance of the wire, and the non diagonal element $Z_{i k}$ represents the mutual impedance; [I] is the load current of the conductor and the ground induced current matrix.

The paper analyzes the calculation methods of ground induced voltage, circulation and energy loss by the example about GJ ground segment insulation、 first terminal grounding 、OPGW tower grounding. The equivalent network of ground induced voltage and circulation is shown in Figure 3. $Z_{L i}$ is the impedance of ground wire from tower $\mathrm{i}$ to tower $\mathrm{i}+1, R_{g i}$ is the grounding resistance of tower I, $U_{i(i+1)}$ is the electromagnetic induction voltage on the ground wire between towers and towers. $1,2, \ldots$ $\mathrm{K}, \ldots \mathrm{N}$ is the tower number, in which $1 \sim \mathrm{k}$ is the tower number in each insulation section, and $1 \sim \mathrm{n}$ is the tower number of the whole line.

According to equation (8), if $i_{1}=i_{2}=0$, electromagnetic induction voltage $U_{i(i+1)}$ can be calculated in each span on the ground. According to the equivalent calculation chart of OPGW circulation, the paper uses mesh current method to solve OPGW induced circulation. According to figure 3 (below), the mesh current equation can be written as $\left[Z_{g w}\right] \cdot\left[I_{g w}\right]=\left[U_{g w}\right]$.
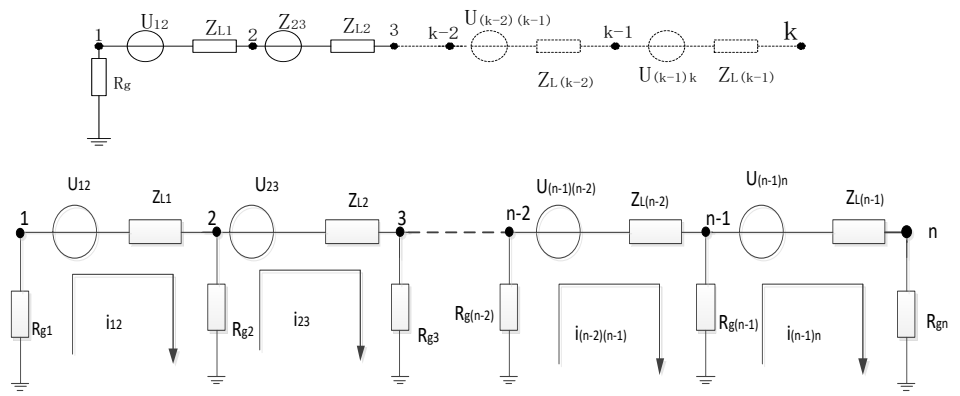

Figure 3. GJ ground induced voltage(top) OPGW circulation(below)

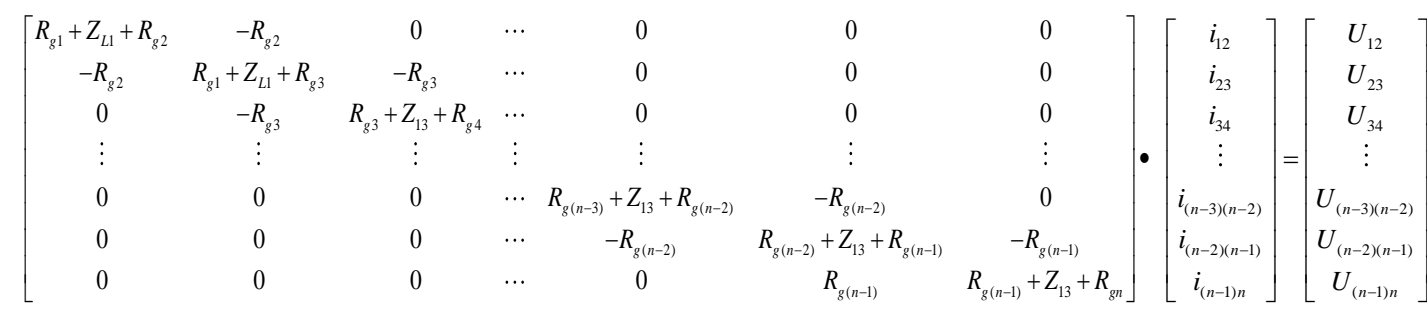

$\left[Z_{g w}\right]$ is the equivalent impedance matrix of OPGW induction loop equivalent calculation network; $\left[I_{g w}\right]$ is the OPGW induced circulation matrix; $\left[U_{g w}\right]$ is the voltage matrix along the OPGW, that is, the tower top potential matrix along the transmission line. The voltage matrix $\left[U_{g w}\right]$ can be obtained by formula (9). $\left[I_{g w}\right]=\left[Z_{g w}\right]^{-1} \cdot\left[U_{g w}\right]$, and the distribution along the OPGW induced 
circulation can be obtained. According to the calculation results of OPGW induced circulation, modified (9) current in the matrix, GJ ground line induced voltage at each spacing can be calculated. According to figure 3 (top), the ground potential within an insulation section from the ground point as i tower:

$U_{1}$ is the pole potential of grounding point.

$$
\phi_{i}=U_{1}+U_{12}+U_{23}+\ldots .+U_{(i-1) i}
$$

In Figure 4: $\mathrm{n}$ is the total number of towers of the whole line, $I_{\text {opgk }}^{2} R_{\text {Lopg }}$ is a the power loss of OPEG in a span; $I_{g k}^{2} R_{L g i}$ is the power loss of ground wire in a span; $I_{g k}^{2} R_{g k}$ is the power loss of ground resistance of $\mathrm{K}$ base tower.

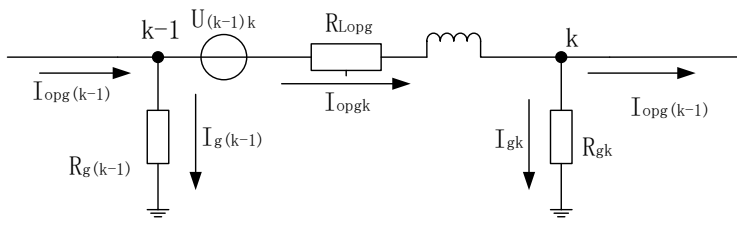

Figure 4. The OPGW equivalent parameters in a span

\section{Calculation description}

According to the operating characteristics of the $750 \mathrm{kV}$ transmission system, a dual power system model consisting of a transmission line and two equivalent power supplies is presented in this paper. In figure 5: $E_{1} 、 E_{2}$ are equivalent power supplies at both ends of the line; $Z_{s 1} 、 Z_{s 2}$ is the equivalent power impedance; $\mathrm{K} 1$ and $\mathrm{K} 2$ are line breakers; $U_{1} 、 U_{2}$ is the bus voltage at both ends of the line; $L_{1} 、 L_{2}$ is the shunt high-voltage reactor for both ends of the line; MOA is line outgoing arrester. Intermediate Line is the main body of $750 \mathrm{kV}$ dual transmission line, and the transmission power of each transmission line is about $2500 \mathrm{MW}$.

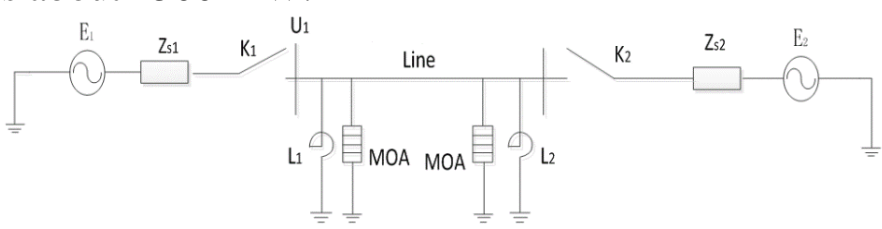

Figure 5. Dual power system model

The transmission line of each span equivalent to $\pi$ type circuit network, a plurality of $\pi$ series circuit to simulate the entire transmission line. The total length of the line is $180 \mathrm{~km}$, and use the reverse phase sequence, reverse phase and full cycle transposition are strictly adopted in the 1/6:1/3:1/3:1/6. The tower of the line is ZGU115 type tower, and its structure size and ground wire number are shown in Figure 6 . The parameters of a transmission line are generally considered to be uniformly distributed along the length, the parameter per unit length is the resistance $r_{0}$, inductance $L_{0}$, conductance $\mathrm{g}_{0}$, Capacitance $C_{0}$.

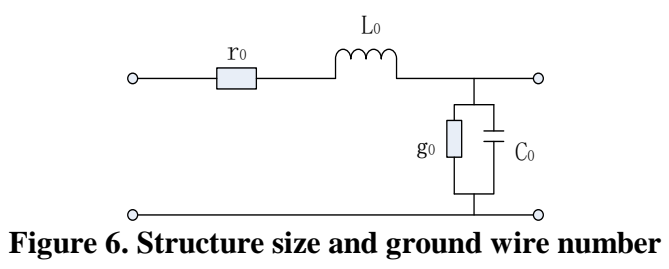

The transformer at both ends of the equivalent system is connected by YNa0d11. The line compensation degree of the model is $81 \%$, and the average distribution is at both ends of the line. The reactance value of the parallel reactor is 3122.2 , and the neutral reactance is configured according to the actual circuit, and the reactance value is 1114 . The calculation results are shown in table 1: 
Table 1. Calculation result

\begin{tabular}{|c|c|c|c|c|}
\hline & \multicolumn{2}{|c|}{ state parameter } & \multicolumn{2}{|c|}{ Parameter quantity } \\
\hline Direct current /A & 0.001 & 1 & 918.75 & 994.54 \\
\hline Power frequency current (p. m)/A & 100 & 500 & 1109.56 & 1158.66 \\
\hline $\begin{array}{c}45 / 90 \mu \mathrm{s} \text { switching impulse } \\
\text { current (p.m)/A }\end{array}$ & 1000 & 2000 & 1200.11 & 1234.00 \\
\hline
\end{tabular}

\section{References}

[1] Liu Zhenya. Ultra-high Voltage Network[M]. Beijing: China Economic Publishing House, 2005.

[2] Shu Yinbiao. Research and application of 1000kV AC UHV transmission technology[J]. Power grid technology, 2005, 29(19):T1-T6.

[3] Chen Weixian, Chen He. Controllable adjustment of shunt reactor[J]. High voltage technology, 2000, 26(5):11-15.

[4] Du Ning. Research on series parallel compensation scheme of UHV transmission line[D]. Baoding: North China Electric Power University, 2005.

[5] Chen Yong, Wan Qifa, et al. Corona characteristics of $1000 \mathrm{kV}$ AC transmission line conductors[J]. Power system technology, 2007, 31 (19): 1-8.

[6] Zeng Qingyu. Study on the electrical and corona characteristics of UHV transmission lines [J]. Power system technology, 2007, 31 (19): 1-8.

[7] Du Binxiang, Zhang Youpeng, Tian Gang, et al. Study on the compensation degree of UHV controllable shunt reactor [J]. high voltage apparatus, 2010, 46 (11): 5-8.

[8] Gao Guanggan. Calculation of corona losses of AC 500-1100kV transmission lines [J]. High voltage technology, 1985 (1): 72-74.

[9] Yu Yongyuan, Yang Qiwen. Power system analysis [M]. Beijing: China Electric Power Press, 2004.

[10] Edris A A.Series compensation schemes reducing the potential of subsynchronous resonance [ J] . IEEE Transactions PWRS, 1990:210 226.

[11] Readers guide to subsynchronous resonance [ J] .IEEE Committee Report, T ransactions PWRS, 1992. $150 \sim 157$.

[12] Yu Qiuxia, Liao Changchu. Reactive power compensation capacity of EHV long distance transmission line Practical calculation [J]. Relay. 2003, 32 (13): $28 \sim 30$. 